\title{
Optimisation of substrate feeding in shake flask cultures of Pichia pastoris for recombinant protein production Monika Bollok ${ }^{1}$, Maria Ruottinen ${ }^{1}$, Mirja Krause ${ }^{1}$, Antti Vasala1 ${ }^{1}$ Eija- Riitta Hämäläinen ${ }^{1}$, Antje Neubauer ${ }^{2}$, Johanna Myllyharju ${ }^{2}$ and Peter Neubauer*1
}

\author{
Address: ${ }^{1}$ Bioprocess Engineering Laboratory, Dept. Process \& Environm. Engin and ${ }^{2}$ Biocenter Oulu, P.O. Box 4300, University of Oulu, FIN- \\ 90014 Oulu, Finland \\ Email: Peter Neubauer* - peter.neubauer@oulu.fi \\ * Corresponding author
}

from The 4th Recombinant Protein Production Meeting: a comparative view on host physiology

Barcelona, Spain. 2I-23 September 2006

Published: 10 October 2006

Microbial Cell Factories 2006, 5(Suppl I):P32 doi:I0.II86/1475-2859-5-SI-P32

(C) 2006 Bollok et al; licensee BioMed Central Ltd.

\section{Background}

Pichia pastoris is used as a common host for production of recombinant proteins. Gene expression is mostly controlled by the AOX promoter through methanol addition. The aim of this study was to investigate whether the generally applied methanol addition protocol is optimum and whether the expression in shake flasks can be improved by applying a different feeding scheme.

Therefore we applied the recently described wireless online monitoring wireless system $\left(\right.$ SENBIT $^{\circledast}$ which allows the application of standard sensors such as $\mathrm{pH}$ and $\mathrm{pO}_{2}$ in shake flasks [1]. Moreover, a sensitive sandwich hybridization technology was used for the quantitative analysis of the expression level for process relevant marker genes which to provide data about the physiological state of the cultures and hereby a better understanding of the microbial responses.

\section{Results}

The impact of the feeding protocol was studied in shake flask cultures of Pichia pastoris for recombinant collagen production with methanol as inducer and carbon source. On-line measurement of $\mathrm{pO}_{2}$ revealed that the standard methanol feeding protocol is not favourable. The culture is starved for long times for methanol and also oxygen may be depleted shortly after a methanol pulse. A fed- batch like feeding procedure was developed by applying a computer controlled micropump system for semi-continuous addition of methanol. As a result the amount of collagen was improved. Furthermore, also the expression of collagen prolyl 4-hydroxylase, a collagen modifying enzyme was strongly increased which resulted in collagen of higher stability. The improvement of the culture conditions with the new feeding protocol were verified by semiquantitative analysis of different cellular mRNAs.

\section{Conclusion}

Regular feeding of small amounts of methanol in a semicontinuous way improves the behaviour of recombinant cultures of Pichia pastoris and increased the amount and quality of collagen in our study. We propose that this method is generally favourable for the optimisation of gene expression in Pichia pastoris shake flask cultures.

\section{Acknowledgements}

This study was supported by the TEKES "Neobio" programme and a grant to MB by the Academy of Finland.

\section{References}

I. Vasala A, Panula J, Bollok M, Illman L, Hälsig C, Neubauer P: A new wireless system for decentralised measurement of physiological parameters from shake flasks. Microb Cell Fact 2006, 5:8. 